CFTP /09-16

IFIC/09-08

\title{
Flavour violation at the LHC: type-I versus type-II seesaw in minimal supergravity
}

\author{
J. N. Esteves:* J. C. Romao \\ Departamento de Física and CFTP, Instituto Superior Técnico \\ Av. Rovisco Pais 1, 1049-001 Lisboa, Portugal \\ M. Hirsch $\$$ and J. W. F. Valle \\ AHEP Group, Instituto de Física Corpuscular - C.S.I.C./Universitat de València \\ Edificio de Institutos de Paterna, Apartado 22085, E-46071 València, Spain \\ W. Porod** \\ Institut für Theoretische Physik und Astronomie, Universität Würzburg \\ Am Hubland, 97074 Wuerzburg
}

\begin{abstract}
We reconsider the role that the possible detection of lepton flavour violating (LFV) decays of supersymmetric particles at the Large Hadron Collider (LHC) can play in helping reconstruct the underlying neutrino mass generation mechanism within the simplest high-scale minimal supergravity (mSUGRA) seesaw schemes. We study in detail the LFV scalar tau decays at the LHC, assuming that the observed neutrino masses arise either through the pure type-I or the simpler type-II seesaw mechanism. We perform a scan over the mSUGRA parameter space in order to identify regions where lepton flavour violating decays of $\chi_{2}^{0}$ can be maximized, while respecting current low-energy constraints, such as those coming from the bounds on $\operatorname{Br}(\mu \rightarrow e \gamma)$. We estimate the cross section for $\chi_{2}^{0} \rightarrow \chi_{1}^{0}+\tau+\mu$. Though insufficient for a full reconstruction of the seesaw, the search for LFV decays of supersymmetric states at the LHC brings complementary information to that coming from low energy neutrino oscillation experiments and LFV searches.
\end{abstract}

Keywords: supersymmetry; neutrino masses and mixing; LHC; lepton flavour violation

${ }^{*}$ Electronic address: joaomest@cftp.ist.utl.pt

${ }^{\dagger}$ Electronic address: jorge.romao@ist.utl.pt

${ }^{\ddagger}$ Electronic address: albert@cftp.ist.utl.pt

$\S$ Electronic address: mahirsch@ific.uv.es

『Electronic address: valle@ific.uv.es

**Electronic address: porod@physik.uni-wuerzburg.de 


\section{INTRODUCTION}

Neutrino oscillation experiments [1] have provided the first signal of physics beyond the standard model (SM). These measurements show that (a) neutrinos have a non-zero mass and (b) lepton flavour is violated. So far there is no experimental data that indicates that lepton number is also broken. However, one expects neutrinos to be Majorana particles, their mass at low energy being described by a unique $(\Delta L=2)$ dimension-5 operator [2]

$$
\mathcal{L}_{m_{\nu}}=\frac{f}{\Lambda}(H L)(H L) .
$$

where $\Lambda$ is some high energy scale, $f$ a dimensionless coupling constant, and $H(L)$ the Higgs boson (lepton) doublets, respectively. Many model realizations of eq. (1) exist, the most famous being the seesaw mechanism. The latter can be implemented via the exchange of a heavy singlet fermion, usually called type-I seesaw [3, [4, 5, 6]. The exchange of a scalar triplet [6, 7, 8, 9, 10, 11] is now known as type-II seesaw ${ }^{1}$. The exchange of a fermionic triplet [12] is called type-III seesaw in [13]. A list of generic 1-loop realizations of eq. (1) can also be found in 13 ]. Further seesaw realizations, such as the inverse and the linear seesaw, are discussed in [14].

At "low" energies one cannot decide whether tree-level or loop physics generates eq. (11), nor can any measurements of neutrino angles, phases or masses distinguish between the different tree-level seesaw realizations. Under the assumption of a pure type-I or pure type-II minimal supergravity seesaw mechanisms, we reconsider here the prospects for reconstructing the underlying high energy parameters from a combination of different measurements. Clearly, observables outside the neutrino sector are needed in order to ultimately learn about the high energy parameters characterizing the seesaw. The classical tree-level realizations of the simplest type-I seesaw mechanism, unfortunately, can not be put to the test in a direct way. This can be straightforwardly understood by inverting eq. (1), which results in $\Lambda \sim f\left(\frac{0.05 \mathrm{eV}}{m_{\nu}}\right) 10^{15} \mathrm{GeV}$.

If the CERN LHC, due to take first data, finds signs of electroweak scale supersymmetry, indirect insight into the high-energy world might become possible through the search for flavour violation effects [15, 16]. Starting from flavour diagonal soft supersymmetry breaking terms at some high energy "unification" scale, flavour violation appears at lower energies due to the renormalization group evolution of the soft breaking parameters [17, 18]. If the seesaw mechanism is responsible for the observed neutrino masses, the neutrino Yukawa couplings leave their imprint in the slepton mass matrices as first shown in [19]. Potentially large LFV is then induced by the flavour off-diagonal structure in the Yukawa couplings required by the large mixing angles observed in oscillation experiments [20]. Expectations for LFV decays such as $l_{i} \rightarrow l_{j}+\gamma$ and $l_{i} \rightarrow 3 l_{j}$ in the supersymmetric seesaw have been studied in [21, 22, 23, 24, 25, 26]. For the related process of $\mu-e$ conversion in nuclei see, for example [27, 28]. The potential of LHC experiments in probing the allowed seesaw parameters

\footnotetext{
${ }^{1}$ This is the opposite convention to that used in [6].
} 
through measurements of masses and branching ratios of supersymmetric particles has been also discussed in Refs. [29, 30, 31, 32, 33].

In two previous studies [34, 35] we have pointed out that ratios of branching ratios are especially useful for learning about the unknown seesaw parameters. In [34] the case of type-I seesaw was discussed, whereas [35] addresses the case of seesaw type-II. For the typeI seesaw, there are in general too many unknown parameters that preclude making any definite predictions for LFV decays. In contrast, in the simplest type-II seesaw model (with only one triplet coupling to standard model leptons) neutrino mixing angles can be related to ratios such as $\operatorname{Br}\left(\tilde{\tau}_{2} \rightarrow e \chi_{1}^{0}\right) / \operatorname{Br}\left(\tilde{\tau}_{2} \rightarrow \mu \chi_{1}^{0}\right)$.

It has been shown that, to a good approximation, such ratios do not depend on the mSUGRA parameter values. However, from an experimental point of view, calculations of absolute event rates are needed, before ratios of different final state channels can be studied. In [34, 35] we took as reference just a few benchmark mSUGRA points, for which we have made detailed studies. In the present paper we calculate branching ratios and event rates over a large region of mSUGRA parameter space, in order to identify the maximal number of events one can expect in experiments at the LHC, while still respecting all low-energy constraints.

The rest of the paper is organized as follows. In the next section we give a short summary of the theoretical setup. In section III we describe our numerical procedure and present our results. Finally we close in section IV with a discussion and a short summary.

\section{THEORY SETUP}

In order to fix the notation, we will briefly discuss the main features of the seesaw mechanism and mSUGRA. The type-I supersymmetric seesaw consists in extending the particle content of the MSSM by three gauge singlet "right-handed" neutrino superfields. The leptonic part of the superpotential is then

$$
W=Y_{e}^{j i} \widehat{L}_{i} \widehat{H}_{d} \widehat{E}_{j}^{c}+Y_{\nu}^{j i} \widehat{L}_{i} \widehat{H}_{u} \widehat{N}_{j}^{c}+M_{i} \widehat{N}_{i}^{c} \widehat{N}_{i}^{c}, \quad i, j=1, \ldots, 3
$$

where $Y_{e}$ and $Y_{\nu}$ denote the charged lepton and neutrino Yukawa couplings, while $\widehat{N}_{i}^{c}$ are the "right-handed" neutrino superfields with $M_{i}$ Majorana mass terms. One can always choose a basis in which the Majorana mass matrix of the "right-handed" neutrinos is brought to diagonal form $\hat{M}_{R}=\operatorname{diag}\left(M_{1}, M_{2}, M_{3}\right)$. Without loss of generality we will also assume that eq. (2) is written in the basis where the charged lepton Yukawa matrix is already diagonal. In this simple setup, the type-I seesaw model, as defined by eq. (21), is characterized by a total of 21 parameters, from which only 12 are measurable in the low-energy theory, as we discuss below.

The effective mass matrix of the "left-handed" neutrinos at low energies is then given as

$$
m_{\nu}=-\frac{v_{u}^{2}}{2} Y_{\nu}^{T} \cdot \hat{M}_{R}^{-1} \cdot Y_{\nu}
$$


so that, for each "right-handed" neutrino, there is one non-zero eigenvalue in $m_{\nu}$. In eq. (3) we use the notation $\left\langle H_{u, d}\right\rangle=\frac{v_{u, d}}{\sqrt{2}}$ for the vacuum expectation values of the neutral components of the Higgs boson doublets.

The parameters of eq.(2) are defined at the Grand Unified Theory (GUT) scale, whereas the entries of eq. (3) are measured at low energies. In order to connect these two scales we numerically solve the full set of renormalization group equations (RGE) [22, 36].

Being complex symmetric, the light Majorana neutrino mass matrix in eq. (3), is diagonalized by a unitary $3 \times 3$ matrix $U$ [6]

$$
\hat{m}_{\nu}=U^{T} \cdot m_{\nu} \cdot U \text {. }
$$

Inverting the seesaw equation, eq. (3), allows to express $Y_{\nu}$ as [37]

$$
Y_{\nu}=\sqrt{2} \frac{i}{v_{u}} \sqrt{\hat{M}_{R}} \cdot R \cdot \sqrt{\hat{m}_{\nu}} \cdot U^{\dagger},
$$

where $\hat{m}_{\nu}$ is the diagonal matrix with $m_{i}$ eigenvalues and in general $R$ is a complex orthogonal matrix. Note that, in the special case $R=1, Y_{\nu}$ contains only "diagonal" products $\sqrt{M_{i} m_{i}}$. In this simplified case the 18 parameters in $Y_{\nu}$ are reduced to 12 . Note that in general type-I seesaw schemes, the unitary matrix diagonalizing the effective neutrino mass matrix differs from the lepton mixing matrix by terms of order $D / M_{R}$, where the $D=Y_{\nu} v_{u}$. For the high-scale schemes considered here one can safely neglect these deviations ${ }^{2}$. In this case we can set the diagonalization matrix as the lepton mixing matrix (partially) determined in neutrino oscillation measurements.

Implementing the type-II seesaw mechanism within supersymmetry requires at least two $S U(2)$ triplet states $T_{1,2}$. A scalar triplet with mass below the GUT scale changes the running of $g_{1}$ and $g_{2}$ in an unwanted way and gauge coupling unification is lost. If instead one adds only complete $S U(5)$ multiplets (or GUT multiplets which can be decomposed into complete $S U(5)$ multiplets) to the standard model particle content, the scale where couplings unify remains the same (at one loop level), only the value of the GUT coupling itself changes [38].

Our numerical calculation uses an $S U(5)$ inspired model [39], which adds a pair of $\mathbf{1 5}$ and $\overline{\mathbf{1 5}}$ to the Minimal Supersymmetric Standard Model (MSSM) particle spectrum. This variant of the type-II seesaw mechanism, as discussed above, allows us to maintain gauge coupling unification even for $M_{T} \ll M_{G}, M_{G}$ being the unification scale. Under $S U(3) \times$ $S U_{L}(2) \times U_{Y}(1)$ the $\mathbf{1 5}$ decomposes as

$$
\begin{aligned}
\mathbf{1 5} & =S+T+Z \\
S & \sim\left(6,1,-\frac{2}{3}\right), \quad T \sim(1,3,1), \quad Z \sim\left(3,2, \frac{1}{6}\right) .
\end{aligned}
$$

\footnotetext{
${ }^{2}$ However for other type-I schemes, like the inverse seesaw [26, 28] this approximation fails and leads to large LFV from right-handed neutrino exchange, even in the absence of supersymmetric contributions. For a systematic perturbative seesaw diagonalization method that covers all cases see the second paper in Ref. [6].
} 
$T$ has the correct quantum numbers to generate the dimension-5 operator of eq. (11). The $S U(5)$ invariant superpotential reads

$$
\begin{aligned}
W & =\frac{1}{\sqrt{2}} \mathbf{Y}_{i j}^{15} \overline{\mathbf{5}}_{i} \cdot \mathbf{1 5} \cdot \overline{\mathbf{5}}_{j}+\frac{1}{\sqrt{2}} \lambda_{1} \overline{\mathbf{5}}_{H} \cdot \mathbf{1 5} \cdot \overline{\mathbf{5}}_{H}+\frac{1}{\sqrt{2}} \lambda_{2} \mathbf{5}_{H} \cdot \overline{\mathbf{1 5}} \cdot \mathbf{5}_{H}+\mathbf{Y}_{i j}^{5} \mathbf{1 0}_{i} \cdot \overline{\mathbf{5}}_{j} \cdot \overline{\mathbf{5}}_{H} \\
& +\mathbf{Y}_{i j}^{10} \mathbf{1 0}_{i} \cdot \mathbf{1 0}_{j} \cdot \mathbf{5}_{H}+M_{15} \mathbf{1 5} \cdot \overline{\mathbf{1 5}}+M_{5} \overline{\mathbf{5}}_{H} \cdot \mathbf{5}_{H} .
\end{aligned}
$$

Here, $\overline{\mathbf{5}}=\left(d^{c}, L\right), \mathbf{1 0}=\left(u^{c}, e^{c}, Q\right), \mathbf{5}_{H}=\left(t, H_{2}\right)$ and $\overline{\mathbf{5}}_{H}=\left(\bar{t}, H_{1}\right)$. Below the GUT scale, in the $S U(5)$-broken phase, the superpotential contains the terms

$$
\begin{aligned}
& \frac{1}{\sqrt{2}}\left(Y_{T}^{i j} L_{i} T_{1} L_{j}+Y_{S}^{i j} d_{i}^{c} S d_{j}^{c}\right)+Y_{Z}^{i j} d_{i}^{c} Z L_{j}+Y_{d}^{i j} d_{i}^{c} Q_{j} H_{d}+Y_{u}^{i j} u_{i}^{c} Q_{j} H_{u}+Y_{e}^{i j} e_{i}^{c} L_{j} H_{d} \\
& +\frac{1}{\sqrt{2}}\left(\lambda_{1} H_{d} T_{1} H_{d}+\lambda_{2} H_{u} T_{2} H_{u}\right)+M_{T} T_{1} T_{2}+M_{Z} Z_{1} Z_{2}+M_{S} S_{1} S_{2}+\mu H_{d} H_{u} .
\end{aligned}
$$

As long as $M_{Z} \sim M_{S} \sim M_{T}$, gauge coupling unification will be preserved. Note that exact equality is not required for a successful unification. In our numerical studies we have taken into account the different running of these mass parameters.

Integrating out the heavy triplets at their mass scale, the dimension-5 operator of eq. (1) is generated and after electroweak symmetry breaking the resulting neutrino mass matrix can be written as

$$
m_{\nu}=\frac{v_{u}^{2}}{2} \frac{\lambda_{2}}{M_{T}} Y_{T}
$$

As in the case of the type-I seesaw, eq. (9) depends on the energy scale. In order to compute the neutrino mass $m_{\nu}$ measured at low energies, one needs to know $\lambda_{2}, Y_{T}$ and $M_{T}$ as input parameters at the high energy scale. As will be discussed in section III one can use an iterative procedure in order to find the high scale parameters from the low energy measured quantities.

Note that, without loss of generality, we have the freedom to write eqs. (2) and (7) in the basis where the charged lepton mass matrix is diagonal, fitting the corresponding Yukawa couplings so as to reproduce the three measured charged lepton masses. However note that there are important differences between the type-I and type-II seesaw schemes. For example, in contrast to type-I, in a pure type-II seesaw scheme the unitary matrix $U$ that diagonalizes eq. (9) coincides with the lepton mixing matrix studied in neutrino oscillations. Moreover, in sequential type-I seesaw for each "right-handed" neutrino added there is one non-zero light neutrino mass eigenstate ${ }^{3}$. In contrast, in type-II seesaw one can produce three neutrino masses with just one pair of triplet superfields, with only one triplet directly coupling to leptons. This implies that in the minimal type-II seesaw one has less parameters than in the sequential type-I seesaw. Indeed from the 12 parameters in the complex symmetric $Y_{T}$

\footnotetext{
${ }^{3}$ We do not consider here the possibility of having just two right-handed neutrino states in the type-I seesaw, called $(3,2)$ in Ref. [6]. This could well account for the current neutrino data with just 12 parameters, instead of the 18 characterizing the sequential $(3,3)$ seesaw considered here.
} 
matrix, one can remove 3 phases by redefining the charged leptons [6]. In addition, from the 3 complex parameters $\lambda_{1,2}$ and $M_{T}$, one does not enter, as only one of the triplets couples to leptons, and finally, two of the three phases can also be removed by field redefinitions. The net result is that there are only 11 physical parameters governing neutrino physics [35]. This number is substantially smaller than the 18 free parameters describing the simplest type-I seesaw scheme containing three "right-handed" neutrinos [40] ${ }^{4}$.

At low energies a maximum of 9 neutrino parameters can be fixed by measuring lepton properties: 3 neutrino masses, 3 mixing angles and $3 \mathrm{CP}$ phases. Thus from neutrino data only, neither type-I nor type-II seesaw schemes can be completely reconstructed, even in their simplest realizations. However, especially important in the following is the fact that low-energy neutrino angles are directly related to the high-energy Yukawa matrix in the type-II seesaw, whereas no such simple connection exists in the seesaw type-I (see also the discussion in [41]).

As already commented, to a good approximation the lepton mixing matrix may be taken in unitary form, with three mixing angles $\theta_{i j}$, and three physical CP phases $\phi_{i j}[6]$. Of these only the leptonic analogue of the Kobayashi-Maskawa phase $\delta$, taken as the invariant combination $\delta \equiv \phi_{12}-\phi_{13}+\phi_{23}$ would enter the class of LFV processes discussed in this paper, so that we get the standard form,

$$
U=\left(\begin{array}{ccc}
c_{12} c_{13} & s_{12} c_{13} & s_{13} e^{-i \delta} \\
-s_{12} c_{23}-c_{12} s_{23} s_{13} e^{i \delta} & c_{12} c_{23}-s_{12} s_{23} s_{13} e^{i \delta} & s_{23} c_{13} \\
s_{12} s_{23}-c_{12} c_{23} s_{13} e^{i \delta} & -c_{12} s_{23}-s_{12} c_{23} s_{13} e^{i \delta} & c_{23} c_{13}
\end{array}\right)
$$

where $s_{i j} \equiv \sin \theta_{i j}, c_{i j}=\cos \theta_{i j}$. Since no current experiment is sensitive enough to probe leptonic $\mathrm{CP}$ violation we take, for simplicity, $\delta=0$. Neutrino oscillation experiments can be fitted with either a normal hierarchical spectrum $(\mathrm{NH})$, or with an inverted hierarchy (IH) one. If one does not insist in ordering the neutrino mass eigenstates $m_{\nu_{i}}, i=1,2,3$ with respect to increasing mass, the matrix $U$ can describe both possibilities without reordering of angles. In this convention, which we will use in the following, $m_{\nu_{1}} \simeq 0\left(m_{\nu_{3}} \simeq 0\right)$ corresponds to normal (inverse) hierarchy and $s_{12}, s_{13}$ and $s_{23}$ are the angles in both types of spectra. Basically $s_{12}$ is measured in solar + reactor experiments, $s_{23}$ in atmospheric + accelerator experiments and $s_{13}$ is constrained by reactor neutrino oscillation data.

In the general MSSM, LFV off-diagonal entries in the slepton mass matrices involve additional free parameters which arise from the mechanism of supersymmetry breaking. In order to relate LFV in the slepton sector with the LFV encoded in $Y_{\nu}$ or $Y_{T}$ one must assume some particular scheme for supersymmetry breaking. For simplicity and definiteness we will adopt mSUGRA boundary conditions, characterized by four continuous real and one discrete free parameter, usually denoted as

$$
m_{0}, M_{1 / 2}, A_{0}, \tan \beta, \operatorname{Sgn}(\mu)
$$

\footnotetext{
${ }^{4}$ We are treating the three charged lepton masses as experimentally determined parameters.
} 
Here, $m_{0}$ is the common scalar mass, $M_{1 / 2}$ the gaugino mass and $A_{0}$ the common trilinear parameter, all defined at the grand unification scale, $M_{G} \simeq 2 \cdot 10^{16} \mathrm{GeV}$. The remaining two parameters are $\tan \beta=v_{u} / v_{d}$ and the sign of the Higgs mixing parameter $\mu$.

In order to have a qualitative understanding of the magnitudes of the LFV rates we first present approximate leading-log analytical solutions for the renormalization group equations ${ }^{5}$. For the case of type-I seesaw, the LFV elements induced in the charged left-slepton mass matrix by renormalization group evolution can be approximated as [22]

$$
\left(\Delta M_{\tilde{L}}^{2}\right)_{i j}=-\frac{1}{8 \pi^{2}}\left(3 m_{0}^{2}+A_{0}^{2}\right)\left(Y_{\nu}^{\dagger} L Y_{\nu}\right)_{i j},
$$

where $Y_{\nu}$ is given in terms of the neutrino parameters by eq. (5) and the factor $L$ is defined as

$$
L_{k l}=\log \left(\frac{M_{G}}{M_{k}}\right) \delta_{k l} .
$$

Similarly, one can get an analogous approximate expression for the off-diagonal elements of the charged left-slepton mass matrix characterizing LFV in type-II seesaw schemes [39].

\section{NUMERICAL RESULTS}

Due to the non-trivial structure of the neutrino Yukawa matrix $Y_{\nu}$ in eq. (5) and of $Y_{T}$ in eq. (9) for type-I and type-II seesaw, respectively, the slepton mass matrices contain calculable LFV entries [17, 19]. In order to determine their magnitude we solve the complete set of renormalization group equations, given in [22, 36, 39]. All results presented below have been obtained with the lepton flavour violating version of the program package SPheno [43], where the RGEs for the MSSM part are implemented at the 2-loop level. For definiteness we set neutrino mass squared differences to their current best fit values [20] and fix the angles to the Tri-Bi-Maximal (TBM) values [45].

Fixing the values of other mSUGRA parameters, we used SPheno to perform a numerical scan over the $m_{0^{-}} M_{1 / 2}$ plane. For each point in this plane, we adjust the value of $M_{R}\left(M_{T}\right)$ in order to keep the low energy LFV observable $\operatorname{BR}(\mu \rightarrow e \gamma)$ within its present experimental upper bound or within the expected sensitivity of the upcoming experiments [44].

For type-I seesaw our numerical procedure to fit these masses is as follows. As we have already commented, the large number of free parameters characterizing even the simplest type-I seesaw schemes forces us to make simplifying assumptions in inverting the seesaw equation, eq. (5). As a first step we assume degenerate "right-handed" neutrinos and the simplest possible, flavour-blind, structure for the matrix $R$, i.e.

$$
R=1, \quad \hat{M}_{R i j}=M_{R} \delta_{i j} .
$$

\footnotetext{
${ }^{5}$ Note that in the numerical code that leads to the results presented in our plots we have numerically solved the full set of RGEs.
} 
Moreover, we fix the values of the light neutrino masses and Yukawa couplings to reproduce the TBM angle values. In order to determine the resulting LFV observables we numerically integrate the renormalization group equations taking into account the flavour structure of the $Y_{\nu}$ matrix. We integrate out every "right-handed" neutrino and its superpartner at the scale associated to its mass, and calculate the corresponding contribution to the dimensionfive operator which is evolved to the electroweak scale. This way we obtain the exact neutrino masses and mixing angles for this first guess. The difference between the results numerically obtained and the input numbers is then minimized in an iterative procedure until convergence is achieved.

For the type-II seesaw the calculations are performed for the 15-plet case, under the assumption $Y_{Z}=Y_{T}=Y_{S}$ at $M_{G}$, as discussed above, and including the one-loop RGEs for the new parameters in SPheno. For consistency, we have also included 1-loop threshold corrections for gauge couplings and gaugino mass parameters at the scale corresponding to the mass of the triplet, $M_{T}$. The MSSM part is implemented at the 2-loop level and, thus, in principle one should also consistently include the effect of the 15-plets for all parameters at this level. However, as discussed in [35], the correct fit of the neutrino data requires that either the triplet (15-plet) Yukawa couplings are small and/or that $M_{T}$ is close to $M_{G}$, implying that the ratio $M_{T} / M_{G}$ is significantly smaller than $M_{G} / m_{Z}$ and thus one expects only small effects. Inverting the seesaw equation for any fixed value of $\lambda_{2}$ in eq. (91), one can get a first guess of the Yukawa couplings for any fixed values of the light neutrino masses as a function of the corresponding triplet mass. This first guess will not give the correct Yukawa couplings, since the neutrino masses and mixing angles are measured at low energy, whereas for the calculation of $m_{\nu}$ we need to insert the parameters at the high-energy scale. However, we can use this first guess to numerically run the RGEs to obtain the exact neutrino parameters (at low energies) for these input values. The difference between the results obtained numerically and the input can then be minimized in a simple iterative procedure until convergence is achieved. As long as neutrino Yukawas are not large, convergence is reached in a few steps. However, in type-II seesaw schemes, the Yukawa couplings run stronger than in the type-I seesaw, thus our initial guess can sizeable deviate from the exact Yukawa coupling values. Since neutrino oscillation data requires at least one neutrino mass to be larger than about $0.05 \mathrm{eV}$, we do not find any solutions for $M_{T}>10^{15} \mathrm{GeV}$.

Finally, the calculation of cross sections for the production of supersymmetric particles was done using Prospino [46]. The input data was taken from SPheno using the SUSY Les Houches Accord standard format [47].

\section{A. $\operatorname{Br}(\mu \rightarrow e+\gamma)$ for type-I and type-II}

In Fig. 11 and Fig. 2 we show the contours for $\operatorname{Br}(\mu \rightarrow e+\gamma)$ in the $m_{0}, M_{1 / 2}$ plane for pure type-I and type-II seesaw schemes, respectively. On the left panel of Fig. 1 we chose a low

value for $M_{R}=10^{13}$, while on the right panel a value of $10^{14} \mathrm{GeV}$ was chosen. In Fig. 2 the same dependence is shown for the type-II seesaw mass scale $M_{T}$. This shows the dependence 

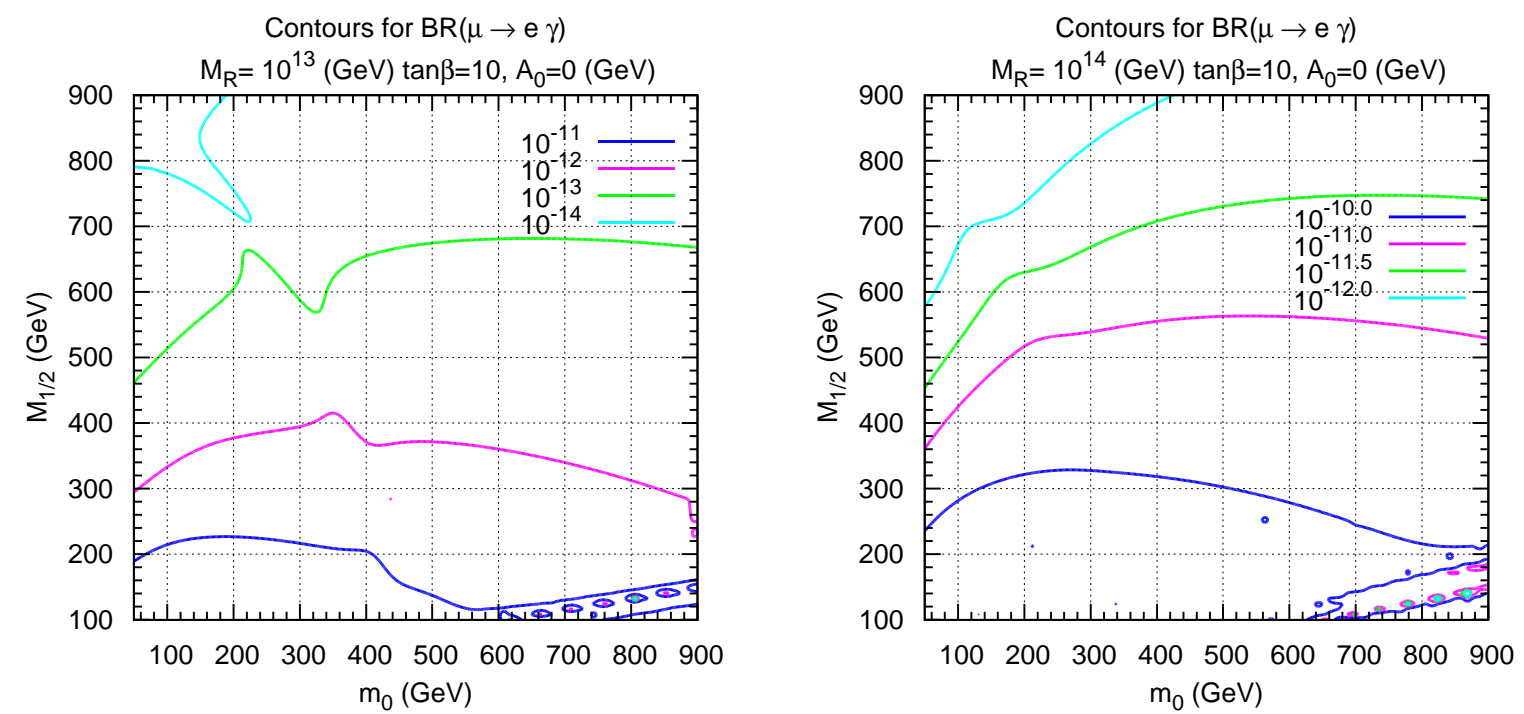

FIG. 1: Contours of $\operatorname{Br}(\mu \rightarrow e+\gamma)$ in the $m_{0}, M_{1 / 2}$ plane for our standard choice of parameters: $\mu>0, \tan \beta=10$ and $A_{0}=0 \mathrm{GeV}$, for type-I with degenerate $\mathrm{RH}$ neutrinos. On the left panel $M_{R}=10^{13} \mathrm{GeV}$, on the right panel $M_{R}=10^{14} \mathrm{GeV}$.
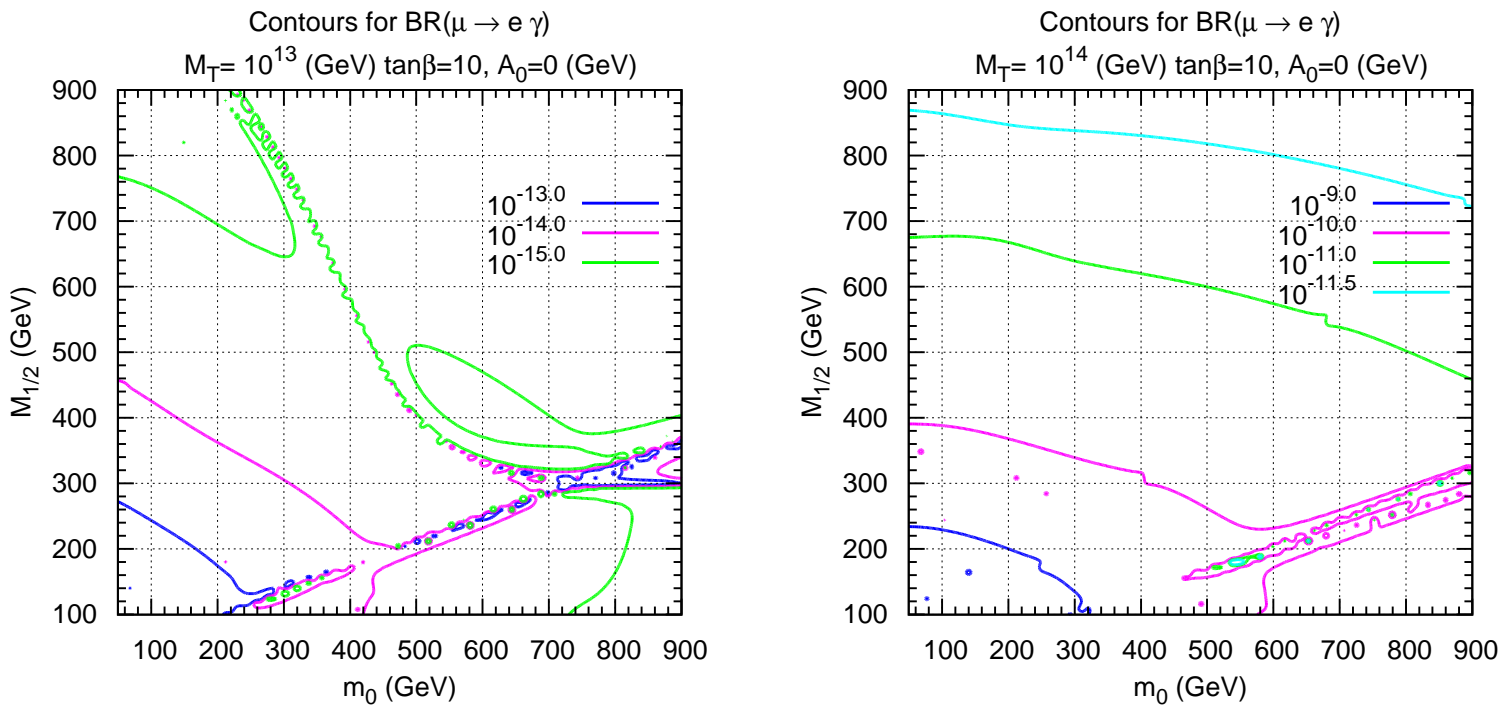

FIG. 2: Contours of $\operatorname{Br}(\mu \rightarrow e+\gamma)$ in the $m_{0}, M_{1 / 2}$ plane for $\lambda_{1}=0.02$ and $\lambda_{2}=0.5$ and for our standard choice of parameters: $\mu>0, \tan \beta=10$ and $A_{0}=0 \mathrm{GeV}$, for type-II seesaw. On the left panel $M_{T}=10^{13} \mathrm{GeV}$, on the right panel $M_{T}=10^{14} \mathrm{GeV}$.

of LFV rates on the choice of scale $M_{R}\left(M_{T}\right)$. The complicated features displayed on these plots are due to cancellations between the chargino and neutralino amplitudes contributing to $\mu \rightarrow e+\gamma$, as is well known [23, 34]. First we note that large parts of parameter space fall within the regions of sensitivity of upcoming experiments like MEG [44]. The contours 
for $\mathrm{BR}(\mu \rightarrow e \gamma)$ are deformed in type-II as compared with respect to those for type-I seesaw. The reason for this is that the addition of gauge non-singlet states in type-II seesaw increases the dependence on the renormalization scale of the neutrino Yukawa coupling and also affects the supersymmetric spectrum, which alters the region where $\operatorname{BR}(\mu \rightarrow e \gamma)$ is strongly suppressed. For our subsequent discussion, the most important point is that for each point in the $m_{0}, M_{1 / 2}$ plane there will be a maximum value of $M_{R}\left(M_{T}\right)$ that will give the maximum possible rates of LFV compatible with current experimental bounds, $\mathrm{BR}(\mu \rightarrow e \gamma)<1.2 \times 10^{-11}$ [42], or with expected sensitivities to be reached at upcoming experiments like MEG, $\operatorname{BR}(\mu \rightarrow e \gamma)<10^{-13}[44]$.

\section{B. LFV stau decays}

The eagerly awaited production of supersymmetric particles at the LHC would open new opportunities for the study of flavour violation in the supersymmetric sector [15]. Here we study how the LFV decays of staus may provide valuable cross-checks of neutrino properties determined at low energies as well as complementary information on the origin of neutrino mass.
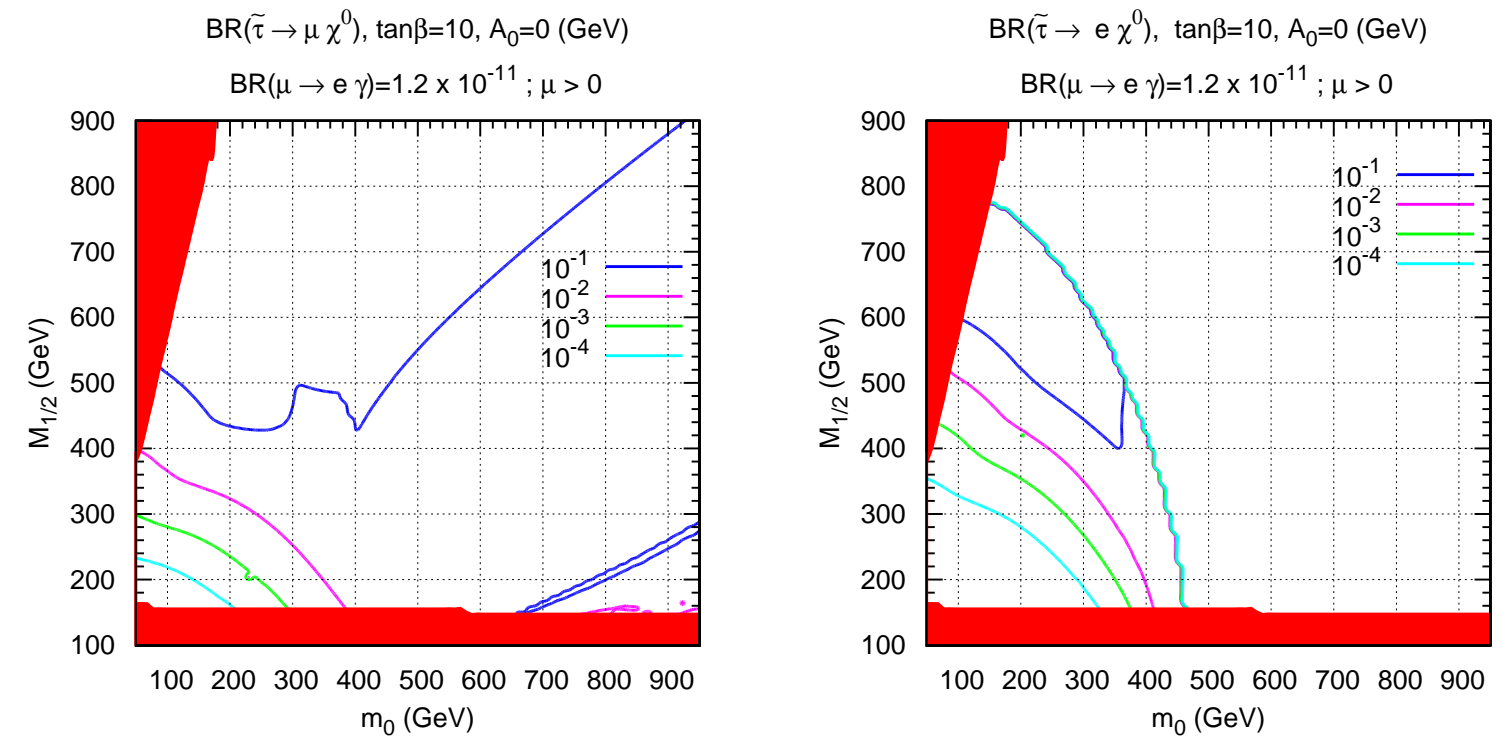

FIG. 3: $\operatorname{Br}\left(\tilde{\tau}_{2} \rightarrow \mu+\chi_{1}^{0}\right)$ (left panel) and $\operatorname{Br}\left(\tilde{\tau}_{2} \rightarrow e+\chi_{1}^{0}\right)$ (right panel), in the $m_{0}, M_{1 / 2}$ plane for our standard choice of parameters: $\mu>0, \tan \beta=10$ and $A_{0}=0 \mathrm{GeV}$, for type-I seesaw, imposing $\operatorname{Br}(\mu \rightarrow e+\gamma) \leq 1.2 \cdot 10^{-11}$.

The expected LFV branching ratios for $\tilde{\tau}_{2} \rightarrow \mu+\chi_{1}^{0}$ and $\tilde{\tau}_{2} \rightarrow e+\chi_{1}^{0}$ depend on the choice of the mSUGRA parameters. After a full scan over the mSUGRA parameter space we found that the dependence on $A_{0}$ and on the sign of $\mu$ is weaker, but that the rates decreased with increasing values of $\tan \beta$. Therefore, we chose our standard point with a relatively low value of $\tan \beta=10$, and for definiteness took $\mu>0$, and $A_{0}=0$. In Fig. 3 

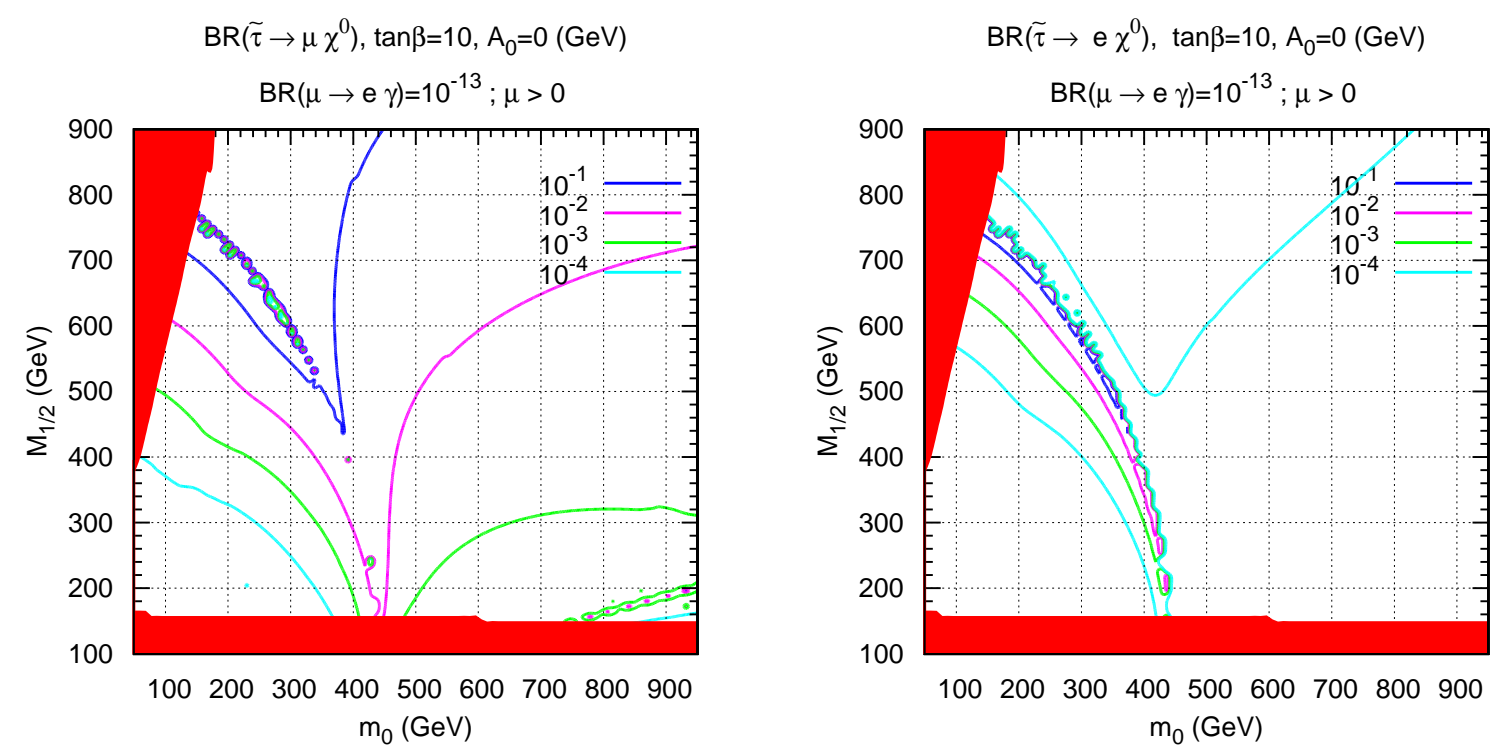

FIG. 4: $\operatorname{Br}\left(\tilde{\tau}_{2} \rightarrow \mu+\chi_{1}^{0}\right)$ (left panel) and $\operatorname{Br}\left(\tilde{\tau}_{2} \rightarrow e+\chi_{1}^{0}\right)$ (right panel), in the $m_{0}, M_{1 / 2}$ plane for our standard choice of parameters: $\mu>0, \tan \beta=10$ and $A_{0}=0 \mathrm{GeV}$, for type-I seesaw, imposing $\operatorname{Br}(\mu \rightarrow e+\gamma) \leq 10^{-13}$

we show the contour plots for the LFV decays $\tilde{\tau}_{2} \rightarrow \mu+\chi_{1}^{0}$ (left panel) and $\tilde{\tau}_{2} \rightarrow e+\chi_{1}^{0}$ (right panel) in the $m_{0}, M_{1 / 2}$ plane for our standard choice of mSUGRA parameters for the simplest pure type-I seesaw scheme. One sees that there are regions in parameter space where the LFV decays of the $\tilde{\tau}_{2}$ can be as large as of order $10^{-1}$. In these plots the values of $M_{R}$ were chosen as to obtain the maximum LFV compatible with the present experimental limit of $\operatorname{Br}(\mu \rightarrow e+\gamma) \leq 1.2 \cdot 10^{-11}$ [42]. Also shown in these plots are the exclusion regions coming from the LEP constraints on SUSY masses and also the exclusion obtained when the neutralino is not the LSP ${ }^{6}$. In Fig. 4 we show the same contour plots for $\operatorname{Br}(\mu \rightarrow e+\gamma) \leq 10^{-13}$, which will be achievable in the coming experiments [44]. Also in this case one observes in Fig. 4 that the LFV stau decay rates may exceed the $10 \%$ level. Notice also that the nontrivial features present in in Figs. 3 and Fig. 4 reflect the well-known cancellations between chargino and neutralino contributions to $\mu \rightarrow e+\gamma$ already discussed above.

In Fig. 5 and Fig. 6 the same type of plots are shown for type-II seesaw. A comparison of these figures shows that, qualitatively, the behavior is very similar for the two types of seesaw. In both cases, the larger rates for $\tilde{\tau}_{2} \rightarrow e+\chi_{1}^{0}$ are more constrained in parameter space than those for $\tilde{\tau}_{2} \rightarrow \mu+\chi_{1}^{0}$. Notice however that there is an important difference between type-I and type-II seesaw, coming from the presence of the Higgs triplets that contribute sizeably to the running of the type-II beta functions. This gets reflected in the supersymmetric particle spectra and hence in the shapes of the red (shaded) regions in

\footnotetext{
${ }^{6}$ Note that we did not display the constraints coming from Dark Matter (DM) relic abundance.
} 
Fig. 5 and Fig. 6. One can observe, indeed, that the regions where the stau is the lightest supersymmetric particle, as well as the regions already excluded by LEP2 are substantially different for type-II seesaw, as compared to the corresponding ones for type-I. This follows from the modification in the beta functions introduced by the addition of the Higgs triplets, making $M_{1}$ and $M_{2}$ smaller in type-II than in type-I seesaw for the same value of $M_{1 / 2}$. The variation with the mSUGRA parameters is illustrated in Fig. 7 (type-I) and Fig. 8 (type-II) for the parameter $A_{0}$ and in Fig. 9 (type-I) and Fig. 10 (type-II) for $\tan \beta$. We can see that there is not much variation with $A_{0}$, while the rates decrease rapidly with increasing values

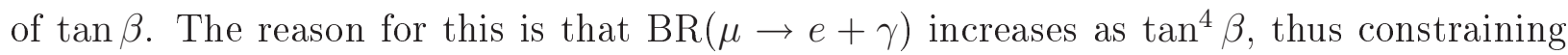
more strongly the maximum attainable stau LFV rates. This effect is stronger for type-I as can be seen by noting the different values for the contour levels in Fig. 9 and Fig. 10, The variation with the sign of $\mu$ is weak and we do not show it here. So, in summary, large LFV rates prefer moderate values of $\tan \beta$ and this explains a posteriori the choice of our standard parameters.
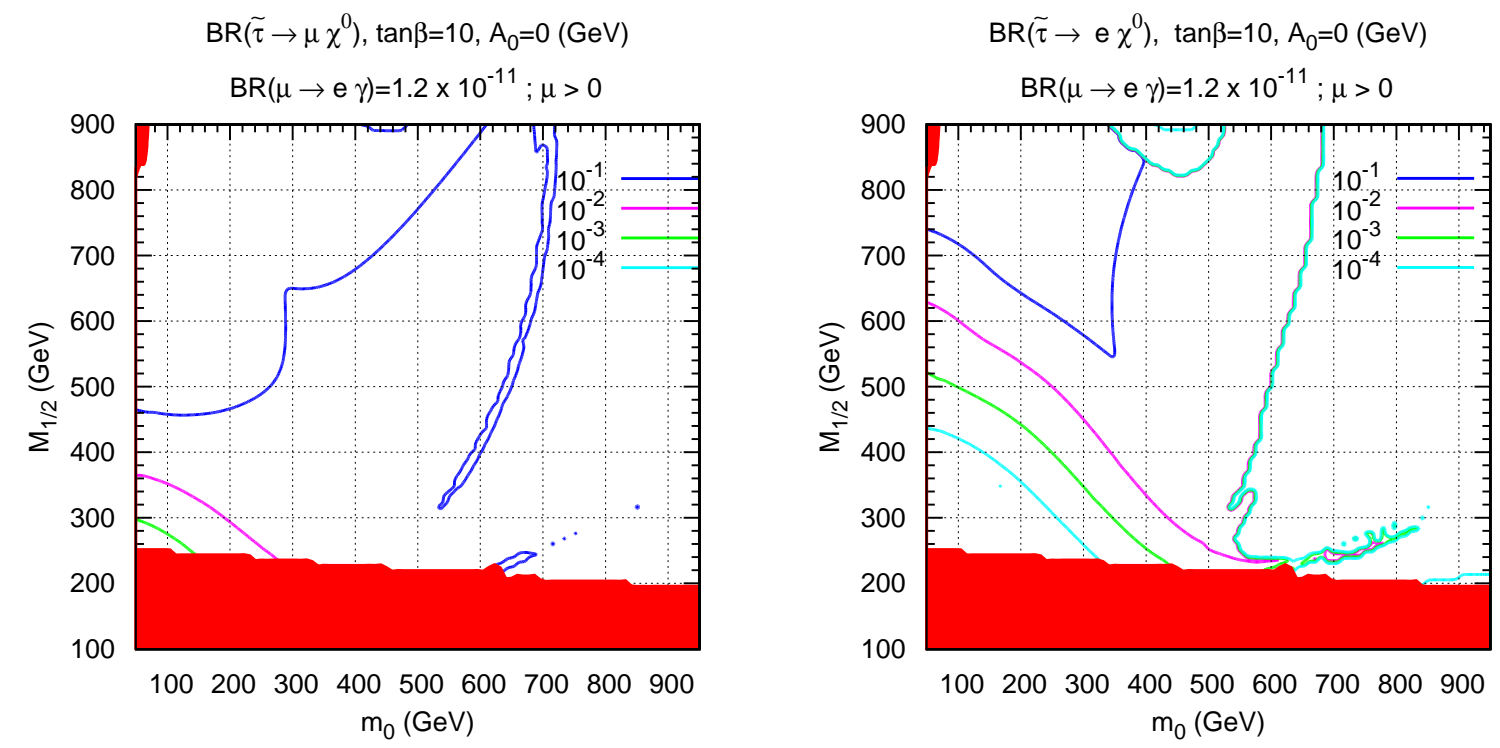

FIG. 5: $\operatorname{Br}\left(\tilde{\tau}_{2} \rightarrow \mu+\chi_{1}^{0}\right)$ (left panel) and $\operatorname{Br}\left(\tilde{\tau}_{2} \rightarrow e+\chi_{1}^{0}\right)$ (right panel), in the $m_{0}, M_{1 / 2}$ plane for $\lambda_{1}=0.02$ and $\lambda_{2}=0.5$ and our standard choice of parameters: $\mu>0, \tan \beta=10$ and $A_{0}=0 \mathrm{GeV}$, for type-II seesaw, imposing $\operatorname{Br}(\mu \rightarrow e+\gamma) \leq 1.2 \cdot 10^{-11}$.

\section{Total production cross section of $\chi_{2}^{0}$}

As important as having a large branching ratio into a LFV final state, is to be able to produce a large enough event sample. In order to estimate the number of LFV events expected at the LHC, one notes that, from Figs. 3] - 10, in the regions where the LFV is sizeable, the direct production of staus at the LHC is negligible compared to that which arises from cascade decays of heavier neutralinos, mainly $\chi_{2}^{0}$. We focus on the $\chi_{2}^{0}$, because decays 

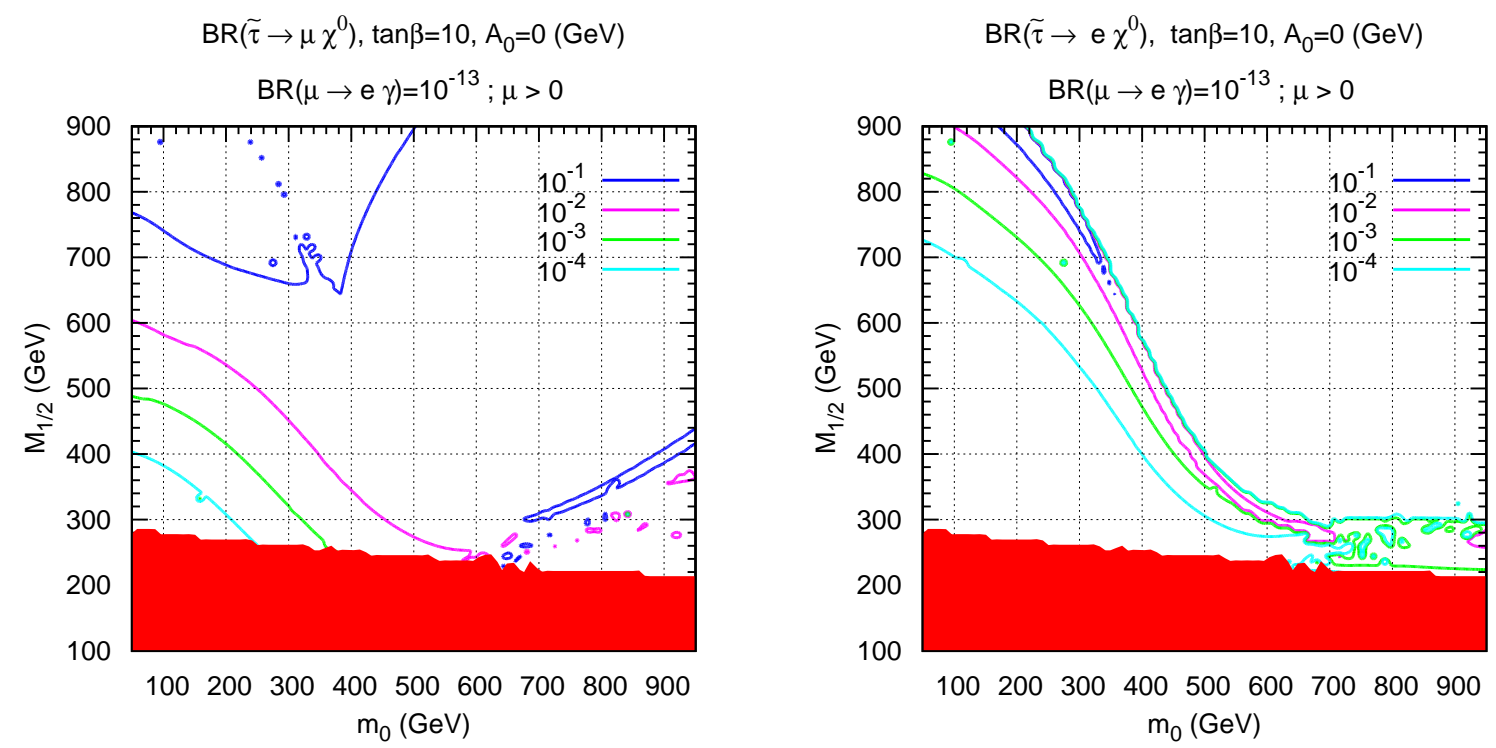

FIG. 6: $\operatorname{Br}\left(\tilde{\tau}_{2} \rightarrow \mu+\chi_{1}^{0}\right)$ (left panel) and $\operatorname{Br}\left(\tilde{\tau}_{2} \rightarrow e+\chi_{1}^{0}\right)$ (right panel), in the $m_{0}, M_{1 / 2}$ plane, for $\lambda_{1}=0.02$ and $\lambda_{2}=0.5$ and our standard choice of parameters: $\mu>0, \tan \beta=10$ and $A_{0}=0 \mathrm{GeV}$, for type-II seesaw, imposing $\operatorname{Br}(\mu \rightarrow e+\gamma) \leq 10^{-13}$.
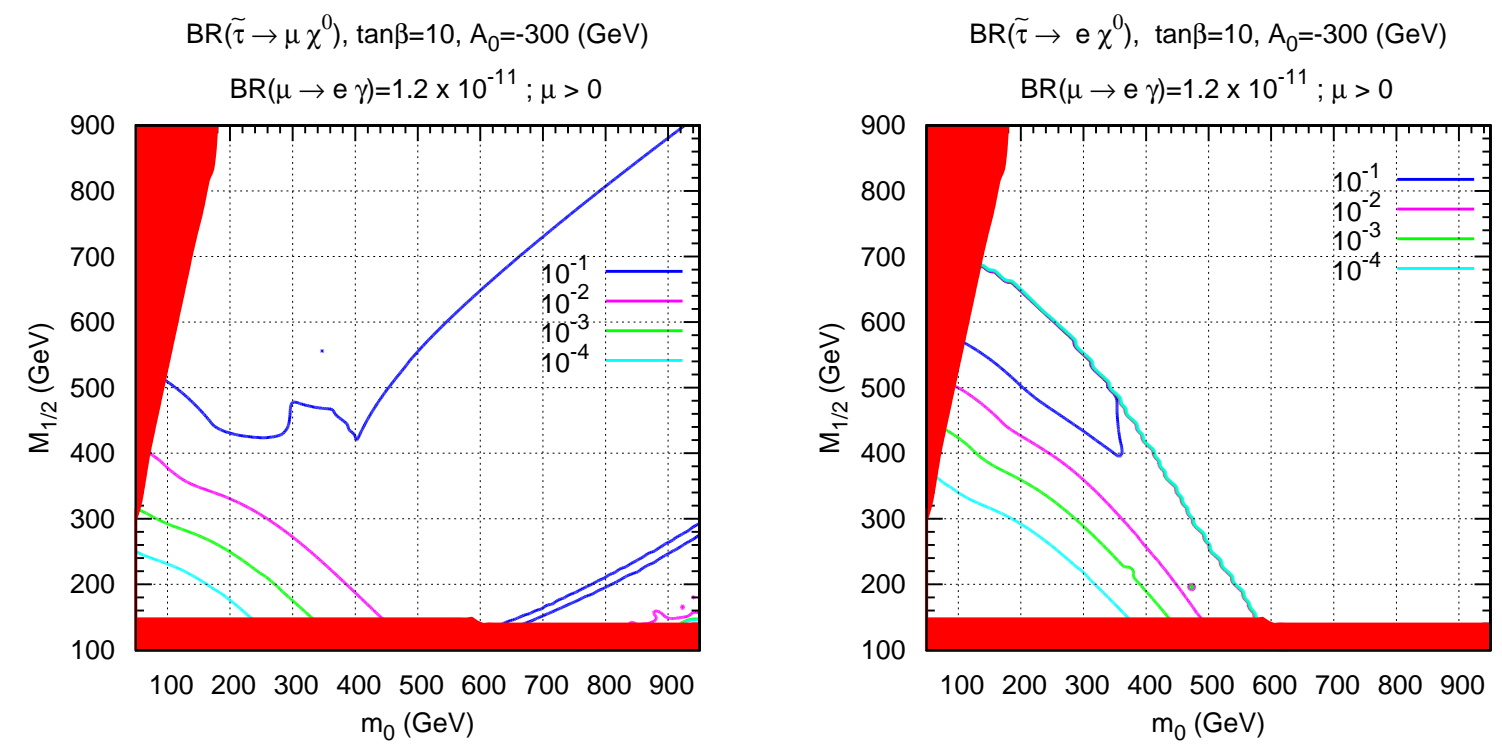

FIG. 7: $\operatorname{Br}\left(\tilde{\tau}_{2} \rightarrow \mu+\chi_{1}^{0}\right)$ (left panel) and $\operatorname{Br}\left(\tilde{\tau}_{2} \rightarrow e+\chi_{1}^{0}\right)$ (right panel), in the $m_{0}, M_{1 / 2}$ plane for standard choice of parameters: $\mu>0, \tan \beta=10$ but different $A_{0}=-300 \mathrm{GeV}$, for type-I seesaw, imposing $\operatorname{Br}(\mu \rightarrow e+\gamma) \leq 1.2 \cdot 10^{-11}$.

such as $\chi_{2}^{0} \rightarrow \mu \tau \chi_{1}^{0}$ are sensitive to flavour violation, whereas in the corresponding chargino decays the flavour information is lost. Hence we first compute the total $\chi_{2}^{0}$ production cross section. In the left panel of Fig. 11] we show the results for the cross section for $\chi_{2}^{0}$ production 

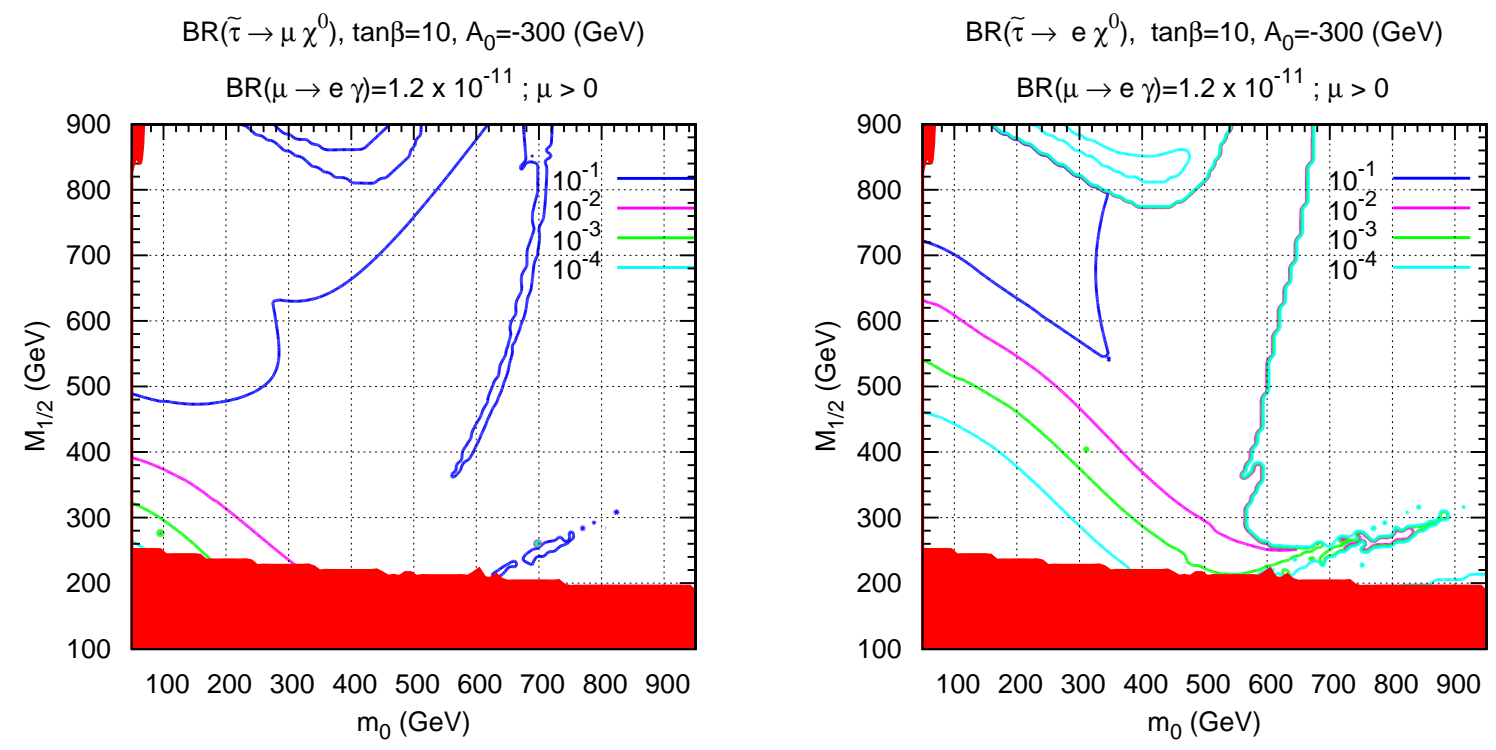

FIG. 8: $\operatorname{Br}\left(\tilde{\tau}_{2} \rightarrow \mu+\chi_{1}^{0}\right)$ (left panel) and $\operatorname{Br}\left(\tilde{\tau}_{2} \rightarrow e+\chi_{1}^{0}\right)$ (right panel), in the $m_{0}, M_{1 / 2}$ plane for $\lambda_{1}=0.02$ and $\lambda_{2}=0.5$ and standard choice of parameters: $\mu>0, \tan \beta=10$ but different $A_{0}=-300 \mathrm{GeV}$, for type-II seesaw, imposing $\operatorname{Br}(\mu \rightarrow e+\gamma) \leq 1.2 \cdot 10^{-11}$.
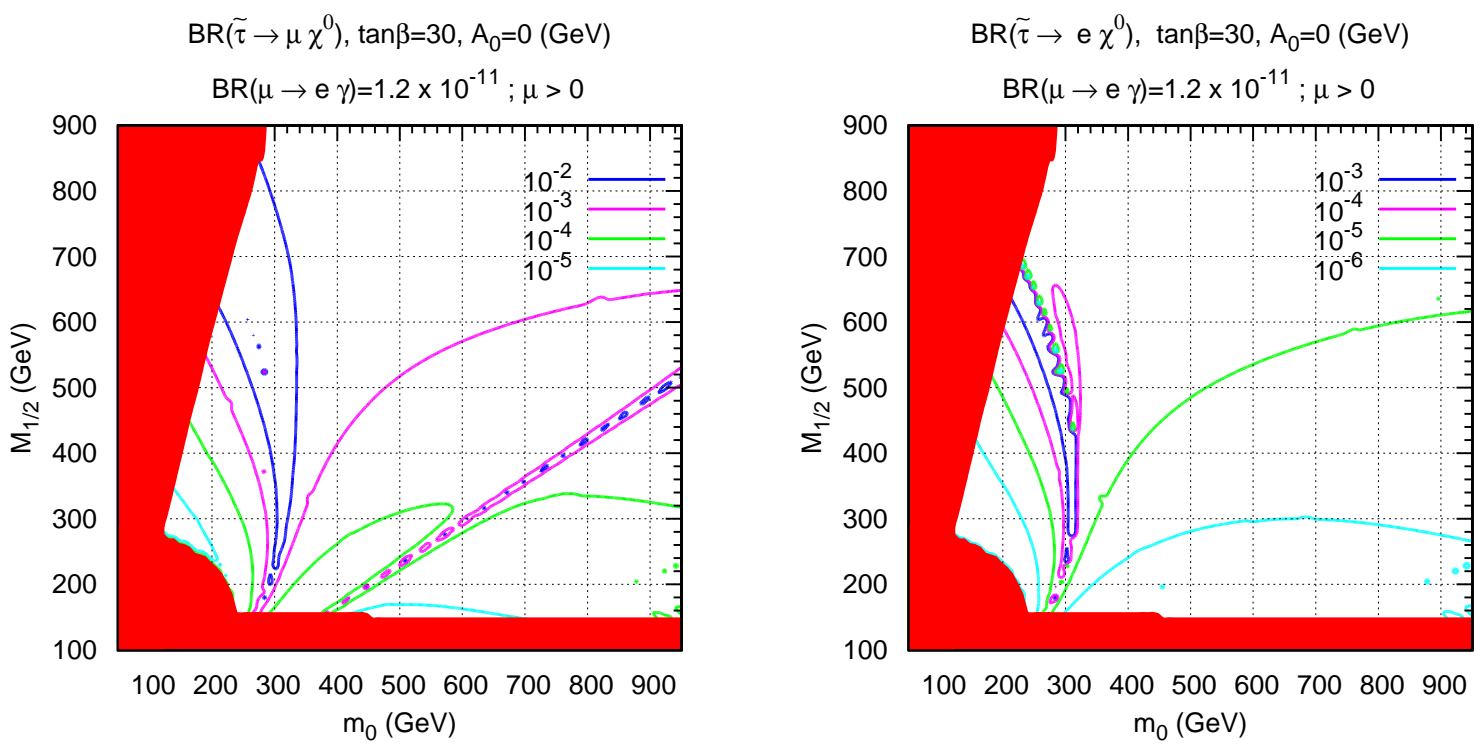

FIG. 9: $\operatorname{Br}\left(\tilde{\tau}_{2} \rightarrow \mu+\chi_{1}^{0}\right)$ (left panel) and $\operatorname{Br}\left(\tilde{\tau}_{2} \rightarrow e+\chi_{1}^{0}\right)$ (right panel), in the $m_{0}, M_{1 / 2}$ plane for standard choice of parameters: $\mu>0, A_{0}=0$ but different $\tan \beta=30$, for type-I seesaw, imposing $\operatorname{Br}(\mu \rightarrow e+\gamma) \leq 1.2 \cdot 10^{-11}$.

as a function of $M_{1 / 2}$, for different choices of $m_{0}$ and for our standard choice of mSUGRA parameters: $\mu>0, \tan \beta=10$ and $A_{0}=0 \mathrm{GeV}$, for the pure type-I mSUGRA seesaw scheme. This choice of mSUGRA parameters corresponds, as will be discussed below, to 

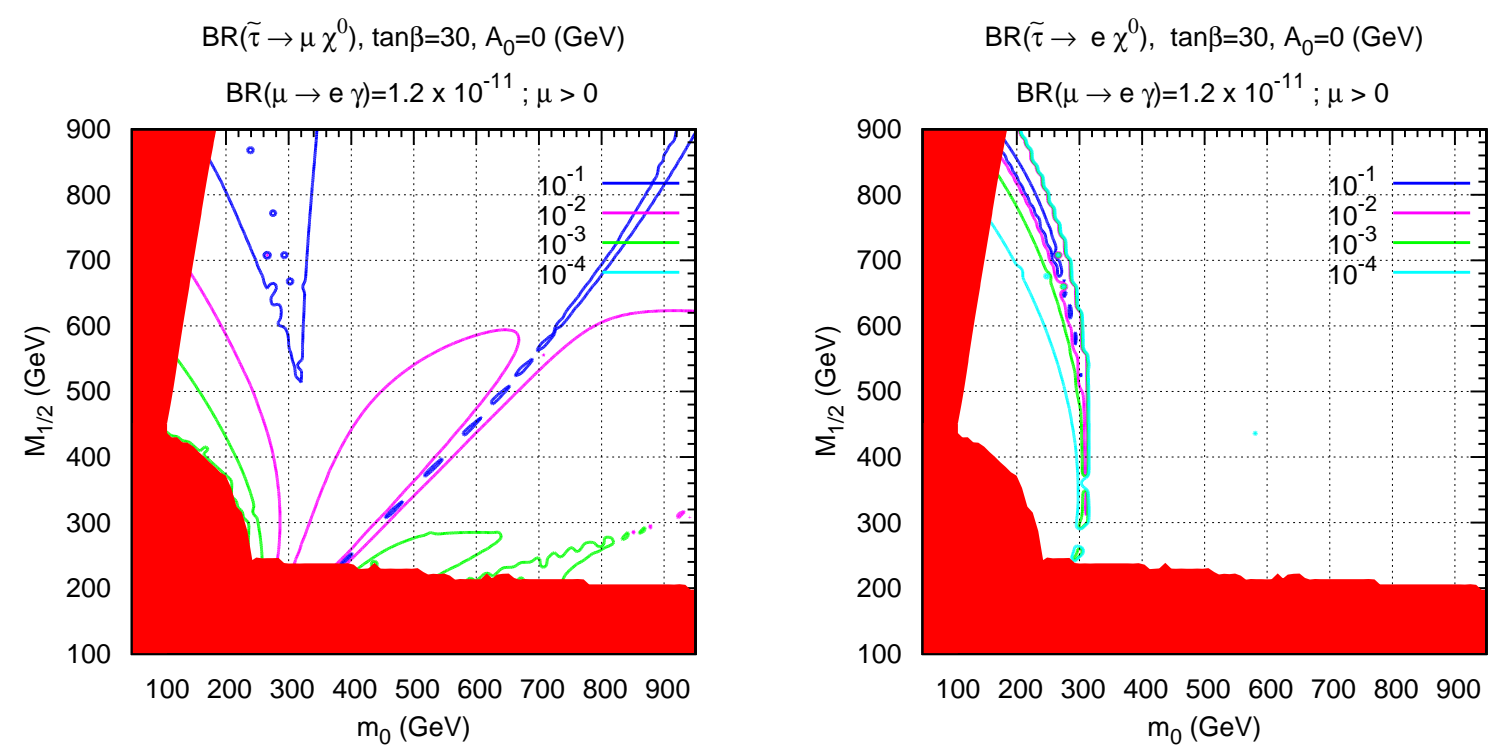

FIG. 10: $\operatorname{Br}\left(\tilde{\tau}_{2} \rightarrow \mu+\chi_{1}^{0}\right)$ (left panel) and $\operatorname{Br}\left(\tilde{\tau}_{2} \rightarrow e+\chi_{1}^{0}\right)$ (right panel), for $\lambda_{1}=0.02$ and $\lambda_{2}=0.5$, in the $m_{0}, M_{1 / 2}$ plane for standard choice of parameters: $\mu>0, A_{0}=0$, but different $\tan \beta=30$, for type-II seesaw, imposing $\operatorname{Br}(\mu \rightarrow e+\gamma) \leq 1.2 \cdot 10^{-11}$.
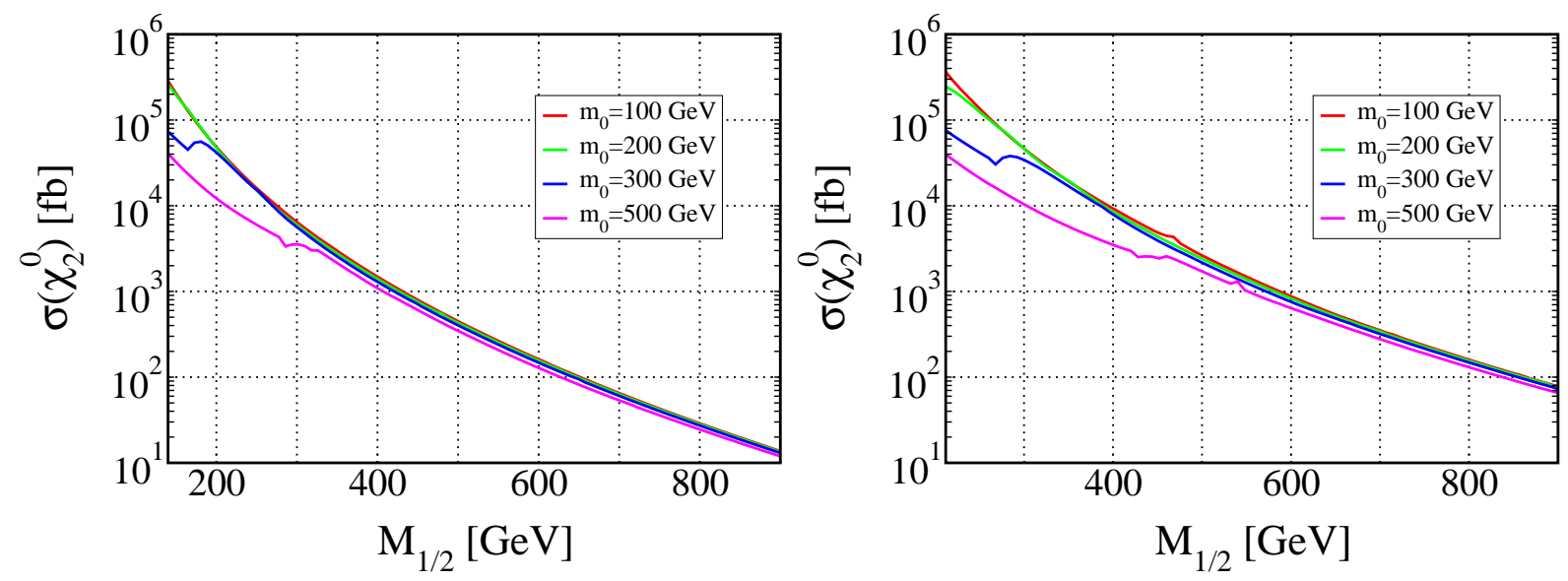

FIG. 11: Production cross section (at leading order) of $\chi_{2}^{0}$ versus $M_{1 / 2}$ for varying $m_{0}$, and for our standard choice of parameters: $\mu>0, \tan \beta=10$ and $A_{0}=0 \mathrm{GeV}$, in type-I seesaw (left panel) and type-II seesaw (right panel) for $\lambda_{1}=0.02$ and $\lambda_{2}=0.5$.

the case where the branching ratios of the LFV stau decays are the largest. This result was obtained using the Prospino code [46] at Leading Order (LO) approximation. We have checked that the Next to Leading Order (NLO) calculation only changes the results slightly, due to an appropriate choice of the renormalization scale [46]. So, in all cross sections presented here, we only used the LO approximation. The corresponding results for type-II seesaw are shown in the right panel of Fig. 11, for the same choice of mSUGRA parameters and for $\lambda_{1}=0.02$ and $\lambda_{2}=0.5$. 


\section{Total production of $\chi_{2}^{0}$ times BR to $\mu-\tau$ lepton pair}

In order to get an estimate of the expected number of LFV events at the LHC we now use a combination of the Prospino and SPheno codes to evaluate the product of the $\chi_{2}^{0}$ production cross section times the branching ratios into LFV processes. Once we know the luminosity at LHC we can multiply it with the above product to get the number of events.

In Fig. 12, we have plotted, for type-I seesaw (left panel) and type-II (right panel), the production cross section at leading order of the second lightest neutralino $\sigma\left(\chi_{2}^{0}\right)$ times the BR of $\chi_{2}^{0}$ going to the opposite-sign dilepton signal $\chi_{1}^{0} \mu \tau$ as a function of $M_{1 / 2}$, for different values of $m_{0}$. We have fixed the rest of the mSUGRA parameters to our standard mSUGRA point and imposed an upper limit on $\operatorname{Br}(\mu \rightarrow e+\gamma) \leq 1.2 \cdot 10^{-11}$. In type-I seesaw, the number of events of the opposite-sign dilepton signal $\chi_{2}^{0} \rightarrow \chi_{1}^{0} \mu \tau$ can be of the order of $10^{3}$ for $m_{0} \sim 100 \mathrm{GeV}$ and $M_{1 / 2} \sim[450,600] \mathrm{GeV}$, assuming a luminosity $\mathcal{L}=100 \mathrm{fb}^{-1}$. In type-II seesaw, there can be a maximum number of events of the order of $10^{3}$ for $m_{0} \sim 100$ $\mathrm{GeV}$ and $M_{1 / 2} \sim[600,800] \mathrm{GeV}$.
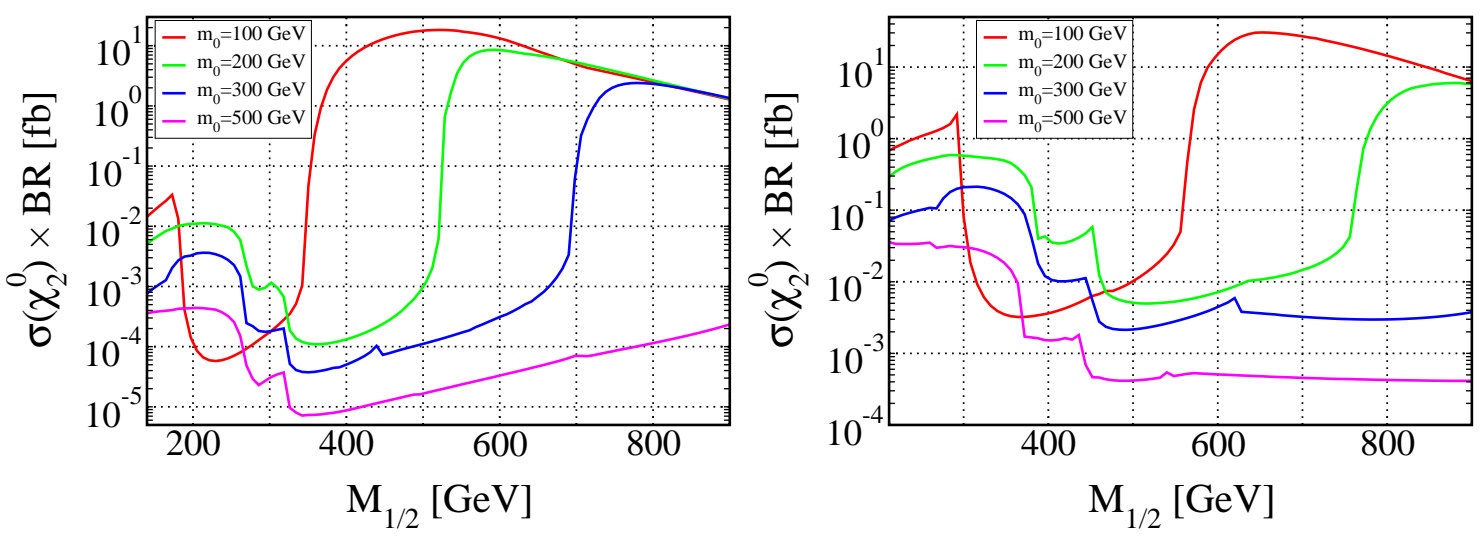

FIG. 12: Production cross section (at leading order) of $\chi_{2}^{0}$ times BR of $\chi_{2}^{0}$ going to $\mu-\tau$ lepton pair versus $M_{1 / 2}$ for $m_{0}=100 \mathrm{GeV}$ (red), $200 \mathrm{GeV}$ (green), $300 \mathrm{GeV}$ (blue) and $500 \mathrm{GeV}$ (magenta), and for our standard choice of parameters: $\mu>0, \tan \beta=10$ and $A_{0}=0 \mathrm{GeV}$, for type-I (left panel) and for type-II seesaw (right panel) with $\lambda_{1}=0.02$ and $\lambda_{2}=0.5$, imposing $\operatorname{Br}(\mu \rightarrow e+\gamma) \leq 1.2 \cdot 10^{-11}$.

For type-II seesaw where we have less parameters, we can look at variations of the result with the values of the triplet Higgs boson coupling $\lambda_{2}$, a parameter that can not be determined from neutrino data alone as it appears only in the ratio $\lambda_{2} / M_{T}$, see eq. (9). In Fig. 13 we show the dependence of the product of cross section times LFV branching ratios as function of $\lambda_{2}$ for our standard point. We should mention that the other Higgs boson triplet coupling $\lambda_{1}$, does not contribute to LFV decays, and hence is left undetermined by this analysis.

As has been discussed in [48], the dominant standard model backgrounds for the process considered are expected to be $W W$ and $t \bar{t}$ production. The cuts necessary to reduce this background will depend on the details of the SUSY spectrum and a detailed investigation is beyond the scope of this paper. The results of [48] suggest that the signal should be visible 


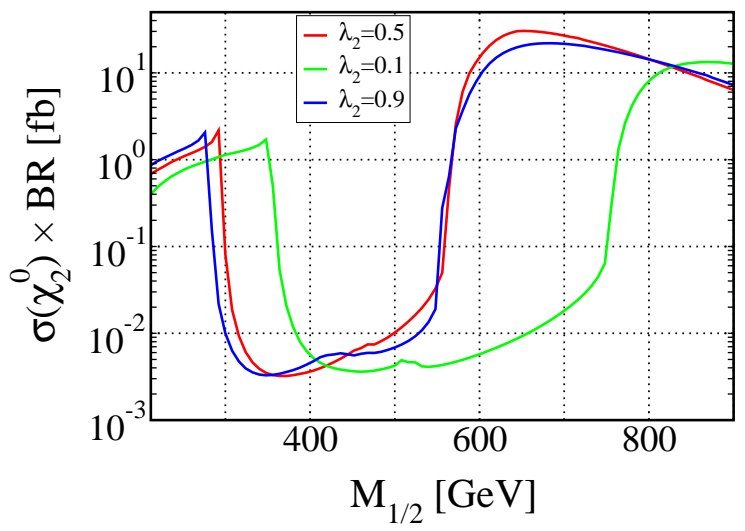

FIG. 13: Production cross section (at leading order) of $\chi_{2}^{0}$ times BR of $\chi_{2}^{0}$ going to $\mu-\tau$ lepton pair versus $M_{1 / 2}$, for our standard choice of parameters: $\mu>0, \tan \beta=10$ and $A_{0}=0 \mathrm{GeV}$, for type-II seesaw, imposing $\operatorname{Br}(\mu \rightarrow e+\gamma) \leq 1.2 \cdot 10^{-11}$, for a fixed value of $m_{0}=100 \mathrm{GeV}$ and different values of $\lambda_{2}=0.1$ (green), 0.5 (red), 0.9 (blue).

for $\sigma\left(\chi_{2}^{0}\right) \times \mathrm{BR}$ of order $\mathcal{O}(10) \mathrm{fb}$.

\section{CONCLUSIONS AND OUTLOOK}

Low energy neutrino experiments, including oscillation studies and neutrinoless doublebeta decay searches may, optimistically, determine at most 9 neutrino parameters: the 3 neutrino masses, the 3 mixing angles and potentially the $3 \mathrm{CP}$ violating phases. This is insufficient to fully reconstruct the underlying mechanism of neutrino mass generation. Under the assumption that neutrino masses arise a la seesaw, we have considered the simplest pure type-I or pure type-II seesaw schemes in mSUGRA.

We have performed a full scan over the mSUGRA parameter space in order to identify regions where LFV decays of $\chi_{2}^{0}$ can be maximal, while still respecting low-energy constraints that follow from the upper bounds on $\operatorname{Br}(\mu \rightarrow e \gamma)$. We have also estimated the expected number of events for $\chi_{2}^{0} \rightarrow \chi_{1}^{0}+\tau+\mu$, for a sample luminosity of $\mathcal{L}=100 \mathrm{fb}^{-1}$. The expected number of events for the other channel $\chi_{2}^{0} \rightarrow \chi_{1}^{0}+\tau+e$ is always smaller, as can be seen from the LVF branching ratios presented in section IIIB. We have found that the pure seesawII scheme is substantially simpler and comes closer to be fully reconstructable, provided additional LFV decays are detected and some supersymmetric particles are discovered at the Large Hadron Collider.

Note that in what concerns the expected maximum number of events both type-I and type-II schemes give similar results. However, as we have seen, given their smaller number of parameters, type-II seesaw schemes are more likely to be reconstructable through a combination of low energy neutrino measurements, with the possible detection of supersymmetric states and lepton flavour violation at the LHC. This should encourage one to perform full-fledged dedicated simulations, in order to ascertain their feasibility within realistic ex- 
perimental conditions [15].

Finally we note that we have not exploited the fact the LFV might induce new "edge variables", giving additional information [49]. We have focused here on LHC, but mention that a future ILC would be much more suited for measuring LFV SUSY processes [29, 50, 51, 52, 53, 54, 55].

\section{Acknowledgements}

Work supported by Spanish grants FPA2008-00319/FPA and Accion Integrada HA-20070090 (MEC). The work of A.V.M. is supported by Fundação para a Ciência e a Tecnologia under the grant SFRH/BPD/30450/2006. The work of J.C.R. and A.V.M is also supported by the RTN Network MRTN-CT-2006-035505 and by Fundação para a Ciência e a Tecnologia through the projects CFTP-FCT UNIT 777, POCI/81919/2007 and CERN/FP/83503/2008. W.P. is partially supported by the German Ministry of Education and Research (BMBF) under contract 05HT6WWA, by the DAAD, project number $\mathrm{D} / 07 / 13468$.

[1] Super-Kamiokande collaboration, Y. Fukuda et al., Phys. Rev. Lett. 81, 1562 (1998), hep-ex/9807003]; Q. R. Ahmad et al. [SNO Collaboration], Phys. Rev. Lett. 89, 011301 (2002); K. Eguchi et al. [KamLAND Collaboration], Phys. Rev. Lett. 90, 021802 (2003).

[2] S. Weinberg, Phys. Rev. Lett. 43, 1566 (1979); S. Weinberg, Phys. Rev. D 22, 1694 (1980).

[3] P. Minkowski, Phys. Lett. B 67 (1977) 421.

[4] T. Yanagida, in KEK lectures, ed. O. Sawada and A. Sugamoto, KEK, 1979; M Gell-Mann, P Ramond, R. Slansky, in Supergravity, ed. P. van Niewenhuizen and D. Freedman (North Holland, 1979).

[5] R.N. Mohapatra and G. Senjanovic, Phys. Rev. Lett. 44912 (1980).

[6] J. Schechter and J. W. F. Valle, Phys. Rev. D 22, 2227 (1980); Phys. Rev. D 25, 774 (1982).

[7] W. Konetschny and W. Kummer, Phys. Lett. B 70, 433 (1977).

[8] R. E. Marshak and R. N. Mohapatra, Invited talk given at Orbis Scientiae, Coral Gables, Fla., Jan 14-17, 1980 (Published in Orbis Scientiae 1980:277).

[9] T. P. Cheng and L. F. Li, Phys. Rev. D 22, 2860 (1980).

[10] G. Lazarides, Q. Shafi and C. Wetterich, Nucl. Phys. B 181, 287 (1981).

[11] R. N. Mohapatra and G. Senjanovic, Phys. Rev. D 23, 165 (1981).

[12] R. Foot, H. Lew, X. G. He and G. C. Joshi, Z. Phys. C 44, 441 (1989).

[13] E. Ma, Phys. Rev. Lett. 81, 1171 (1998) arXiv:hep-ph/9805219.

[14] J. W. F. Valle, J. Phys. Conf. Ser. 53, 473 (2006) arXiv:hep-ph/0608101.

[15] F. del Aguila et al., Eur. Phys. J. C 57 (2008) 183 [arXiv:0801.1800 [hep-ph]].

[16] M. Raidal et al., Eur. Phys. J. C 57, 13 (2008) [arXiv:0801.1826 [hep-ph]]. 
[17] L. J. Hall, V. A. Kostelecky and S. Raby, Nucl. Phys. B267, 415 (1986).

[18] J. F. Donoghue, H. P. Nilles and D. Wyler, Phys. Lett. B 128, 55 (1983).

[19] F. Borzumati and A. Masiero, Phys. Rev. Lett. 57, 961 (1986).

[20] For a review of neutrino oscillation data, see: M. Maltoni, T. Schwetz, M. A. Tortola and J. W. F. Valle, New J. Phys. 6, 122 (2004); an updated analysis has now been published in T. Schwetz, M. Tortola and J. W. F. Valle, New J. Phys. 10, 113011 (2008) arXiv:0808.2016 [hep-ph]] where all experimental references can be found.

[21] J. Hisano, T. Moroi, K. Tobe, M. Yamaguchi and T. Yanagida, Phys. Lett. B357, 579 (1995) hep-ph/9501407.

[22] J. Hisano, T. Moroi, K. Tobe and M. Yamaguchi, Phys. Rev. D53, 2442 (1996) hep-ph/9510309.

[23] F. Deppisch, H. Päs, A. Redelbach, R. Rückl and Y. Shimizu, Eur. Phys. J. C28, 365 (2003) hep-ph/0206122.

[24] E. Arganda and M. J. Herrero, Phys. Rev. D73, 055003 (2006) hep-ph/0510405.

[25] S. Antusch, E. Arganda, M. J. Herrero and A. M. Teixeira, JHEP 11, 090 (2006) hep-ph/0607263.

[26] F. Deppisch and J. W. F. Valle, Phys. Rev. D72, 036001 (2005) hep-ph/0406040.

[27] E. Arganda, M. J. Herrero and A. M. Teixeira, JHEP 10, 104 (2007) [0707.2955].

[28] F. Deppisch, T. S. Kosmas and J. W. F. Valle, Nucl. Phys. B752, 80 (2006) hep-ph/0512360.

[29] J. Hisano, M. M. Nojiri, Y. Shimizu and M. Tanaka, Phys. Rev. D60, 055008 (1999) hep-ph/9808410].

[30] G. A. Blair, W. Porod and P. M. Zerwas, Eur. Phys. J. C27, 263 (2003) hep-ph/0210058.

[31] A. Freitas, W. Porod and P. M. Zerwas, Phys. Rev. D72, 115002 (2005) hep-ph/0509056.

[32] M. R. Buckley and H. Murayama, Phys. Rev. Lett. 97, 231801 (2006) hep-ph/0606088.

[33] F. Deppisch, A. Freitas, W. Porod and P. M. Zerwas, Phys. Rev. D 77, 075009 (2008) arXiv:0712.0361 [hep-ph]].

[34] M. Hirsch, J. W. F. Valle, W. Porod, J. C. Romao and A. Villanova del Moral, Phys. Rev. D 78, 013006 (2008) arXiv:0804.4072 [hep-ph].

[35] M. Hirsch, S. Kaneko and W. Porod, Phys. Rev. D 78, 093004 (2008) arXiv:0806.3361 [hep$\mathrm{ph}]$.

[36] S. Antusch and M. Ratz, JHEP 07, 059 (2002) hep-ph/0203027.

[37] J. A. Casas and A. Ibarra, Nucl. Phys. B618, 171 (2001) hep-ph/0103065.

[38] P. Langacker, Phys. Rept. 72, 185 (1981).

[39] A. Rossi, Phys. Rev. D 66, 075003 (2002) arXiv:hep-ph/0207006; F. R. Joaquim and A. Rossi, Phys. Rev. Lett. 97, 181801 (2006) arXiv:hep-ph/0604083.

[40] A. Santamaria, Phys. Lett. B 305, 90 (1993) arXiv:hep-ph/9302301.

[41] J. R. Ellis, J. Hisano, M. Raidal and Y. Shimizu, Phys. Rev. D 66, 115013 (2002) arXiv:hep-ph/0206110.

[42] C. Amsler et al., Physics Letters B667, 1 (2008).

[43] W. Porod, Comput. Phys. Commun. 153, 275 (2003) arXiv:hep-ph/0301101. 
[44] See, for example, http://meg.icepp.s.u-tokyo.ac.jp/

[45] P. F. Harrison, D. H. Perkins and W. G. Scott, Phys. Lett. B 530, 167 (2002) arXiv:hep-ph/0202074.

[46] W. Beenakker, R. Hopker and M. Spira, arXiv:hep-ph/9611232; W. Beenakker, M. Kramer, T. Plehn, M. Spira and P. M. Zerwas, Nucl. Phys. B 515, 3 (1998) arXiv:hep-ph/9710451; W. Beenakker, M. Klasen, M. Kramer, T. Plehn, M. Spira and P. M. Zerwas, Phys. Rev. Lett. 83, 3780 (1999) [Erratum-ibid. 100, 029901 (2008)] |arXiv:hep-ph/9906298]; M. Spira, arXiv:hep-ph/0211145; T. Plehn, Czech. J. Phys. 55, B213 (2005) arXiv:hep-ph/0410063.

[47] P. Skands et al., JHEP 0407, 036 (2004) arXiv:hep-ph/0311123; B. Allanach et al., Comput. Phys. Commun. 180, 8 (2009) arXiv:0801.0045 [hep-ph]].

[48] E. Carquin, J. Ellis, M. E. Gomez, S. Lola and J. Rodriguez-Quintero, arXiv:0812.4243 [hep$\mathrm{ph}]$.

[49] A. Bartl, K. Hidaka, K. Hohenwarter-Sodek, T. Kernreiter, W. Majerotto and W. Porod, Eur. Phys. J. C 46, 783 (2006) arXiv:hep-ph/0510074.

[50] N. V. Krasnikov, Phys. Lett. B 388, 783 (1996) arXiv:hep-ph/9511464.

[51] N. Arkani-Hamed, H. C. Cheng, J. L. Feng and L. J. Hall, Phys. Rev. Lett. 77, 1937 (1996) arXiv:hep-ph/9603431.

[52] N. Arkani-Hamed, J. L. Feng, L. J. Hall and H. C. Cheng, Nucl. Phys. B 505, 3 (1997) arXiv:hep-ph/9704205.

[53] D. Nomura, Phys. Rev. D 64, 075001 (2001) arXiv:hep-ph/0004256.

[54] W. Porod and W. Majerotto, Phys. Rev. D 66, 015003 (2002) arXiv:hep-ph/0201284.

[55] F. Deppisch, H. Päs, A. Redelbach, R. Rückl and Y. Shimizu, Phys. Rev. D 69, 054014 (2004) arXiv:hep-ph/0310053. 